\title{
C. Mansion, De la penitance Adam
}

\section{Maria Colombo Timelli}

\section{(2) OpenEdition}

\section{Journals}

\section{Édition électronique}

URL : http://journals.openedition.org/studifrancesi/10526

DOI : 10.4000/studifrancesi.10526

ISSN : 2427-5856

\section{Éditeur}

Rosenberg \& Sellier

\section{Édition imprimée}

Date de publication : 1 décembre 2017

Pagination : $532-533$

ISSN : 0039-2944

\section{Référence électronique}

Maria Colombo Timelli, «C. Mansion, De la penitance Adam », Studi Francesi [En ligne], 183 (LXI | III) |

2017, mis en ligne le 01 février 2018, consulté le 22 janvier 2021. URL : http://

journals.openedition.org/studifrancesi/10526 ; DOI : https://doi.org/10.4000/studifrancesi.10526

Ce document a été généré automatiquement le 22 janvier 2021.

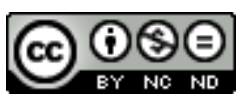

Studi Francesi è distribuita con Licenza Creative Commons Attribuzione - Non commerciale - Non opere derivate 4.0 Internazionale. 


\title{
C. Mansion, De la penitance Adam
}

\author{
Maria Colombo Timelli
}

\section{RÉFÉRENCE}

COLARD MANSION, De la penitance Adam. Texte édité, présenté et annoté par sylviane Messerli d'après les manuscrits Paris, BNF, fr. 1837 et Paris, BNF, Bibliothèque de l'Arsenal, ms. 5092, Paris, Honoré Champion Éditeur, 2016, «Classiques Français du Moyen Âge» 178, 177 pp.

1 Colard Mansion, que la ville de Bruges va célébrer au printemps 2018 par une exposition qui fera date, fut aussi copiste et traducteur du latin; son activité se déploie dans cette même ville, entre les années 1457/58 et 1484, date après laquelle on perd sa trace, et est au moins en partie liée au mécénat de Louis de la Gruthuuse, célèbre bibliophile appartenant à la plus haute aristocratie des Flandres sous les ducs de Bourgogne.

2 Sylviane Messerli offre la première édition de la traduction d'une Vita Adae et Evae, inconnue jusqu'ici, alors que d'autres adaptations de Colard Mansion sont éditées depuis longtemps: la Vie de saint Hubert par Ferdinand C. de Rooy (Zwolle, 1958), le Dialogue des créatures par Pierre Ruelle (Bruxelles, Académie royale de Belgique, 1984), le Donat espirituel par moi-même (Milano, Istituto Lombardo-Accademia di Scienze e Lettere, 1997). La plus importante d'entre elles, à savoir la version en prose des Métamorphoses d'Ovide, dont l'incunable somptueusement illustré fut sans doute la cause de la ruine financière de Mansion, attend en revanche toujours une édition (on verra les deux études de Stefania Cerrito qui ne paraissent pas dans la bibliographie de S.M.: l'une dans «Le moyen français» 69, 2011, pp. 1-14; l'autre dans Pour un nouveau répertoire des mises en prose, Paris, 2014, pp. 85-99).

L'Introduction, pp.9-77, offre les informations essentielles sur le plan historique et littéraire; soulignons d'entrée de jeu l'absence regrettable d'une introduction linguistique (seuls quelques aspects du rapport avec la source latine sont abordés pp. 22-24, alors que des picardismes auraient mérité au moins une remarque: 
phonétique, morphologie, lexique, sont tous concernés). La bibliographie, volontairement sélective (p. 60-65) exclut en particulier les études sur Colard Mansion imprimeur: outre les articles de S. Cerrito à peine mentionnés, il faudra ajouter au moins la synthèse fournie par Renaud Adam («Colard Mansion, passeur de textes?», dans Le roman français dans les premiers imprimés, Paris, 2016, pp. 11-24).

Le titre Penitance Adam couvre en réalité, comme l'explique très clairement l'éditrice, un texte composite; la traduction de la Vita Adae et Evae proprement dite, transmise par trois manuscrits brugeois du dernier quart $\mathrm{du} \mathrm{xv}^{\mathrm{e}}$ siècle (aux deux cités dans le titre de l'édition s'ajoute celui déposé maintenant auprès de la Galerie Les Enluminures, auquel l'éditrice a eu accès), se trouve encadrée, dans le manuscrit de l'Arsenal et dans celui des Enluminures, entre deux autres textes: une "Creation d'Adam» (le titre est moderne), fondée essentiellement sur la Genèse (pp. 40-42), et la Légende du bois de la sainte croix (le texte latin est transmis par plus de quatre-vingts manuscrits, mais le manque d'une édition critique empêche d'en dire plus sur la source de Mansion). Ces trois petits textes portent respectivement sur la création d'Adam et Ève et le péché originel («Creation»), sur leur expulsion du jardin d'Éden et leur vie sur terre, y compris la «penitance» qu'ils assument (Penitance Adam), avec une ouverture sur la rédemption apportée par le Christ (Légende): les échos de l'un à l'autre, nombreux, permettent entre autres une lecture typologique des Écritures.

5 La traduction de la Vita peut être datée inter 1472 et 1484, vraisemblablement ante 1477 (pp. 14-15); sans qu'on puisse la rattacher précisément à un manuscrit latin (il en existe plus de cent), S.M. parvient à l'associer à la rédaction dite «rhénane», bien diffusée dans le nord de l'Europe (pp.37-38). Des questions importantes restent néanmoins ouvertes: il est en effet difficile de savoir si le projet initial de Mansion comprenait l'assemblage des trois textes, ou bien si la "Création» et la Légende constituent des ajouts ultérieurs, et surtout si la traduction de ces deux derniers doit lui être attribuée ou non (pp. 19-21). Ce qui est sûr-S.M. le souligne fort opportunément - c'est que le manuscrit fr. 1837, offert à Louis de Bruges, contient la version la plus proche de la source latine et doit par conséquent constituer le texte de base pour la partie centrale de l'édition (§ 6-22); pour les deux autres parties (§ 1-5 pour la «Création», § 23-33 pour la Légende), il est nécessaire d'avoir recours au manuscrit de l'Arsenal, dont le texte semble moins altéré que celui des Enluminures. Si ce choix n'apparaît nullement contestable, remarquons néanmoins que la transmission reconstruite p.56 (un «original» antérieur au ms fr. 1837, «qui aurait servi tant à $P[=$ fr. 1837] qu'à $A[=$ Arsenal] E [= Enluminures]») me semble ignorer la nécessité de postuler un subarchétype commun justement à $\mathrm{AE}$, au vu des variantes communes de ceux-ci (fautes et lacunes énumérées aux pp. 52-54).

6 L'édition occupe les pp. 79-120; les critères de transcription, énoncés aux pp. 57-60, ne sont pas toujours exactement respectés: la cédille n'est pas utilisée pour les formes du verbes scavoir (sic, par ex. aux pp. 79, 85, 86, 87, 88, 109...), le tréma manque sur certaines formes du verbe oïr (oyans p. 95, oye p. 97, oyez pp. 102, 107, 117, oyant p. 114). Le choix de respecter la segmentation des mots telle qu'elle apparaît dans les manuscrits (p. 59) donne lieu à des alternances inutiles: ainsi l'adverbe plus peut se trouver soudé à l'adjectif qui suit, ou séparé, au sein de la même phrase, voire du même syntagme: «la maison de Nostre Seigneur plushaute et plus excellente que la premiere n'estoit» (p. 101), «[le saint arbre] fut trouvé de tous les aultres le plus court, combien que par la vraye mesure il eust esté de tous les aultres le pluslong» (p.117); le groupe 
prép. + verbe peut être agglutiné (c'est adire p. 85, 109) ou séparé («[Eve] commença $a$ dire» p. 97); pour ce / pource alternent: «et pource [l'homme] est il dit innocent et sans vice [...]; pource qu'il est constitué seigneur de par-dessus toutes aultres creatures ayans ame...» (p. 85); ainsi que pour quoi / pourquoi quel que soit le sens (adverbe interrogatif ou prép. + relatif): «Pourquoy Eve en plourant dist a Adam» (p. 89), «[Seth] commença moult fort a penser pour quoy cest arbre ainsy devenu estoit» (p. 111). Sans nier l'intérêt de ces segmentations (sur lesquelles Nelly Andrieux-Reix et Simone Monsonego ont attiré l'attention depuis vingt ans déjà), on aurait pu les commenter en rapport avec les habitudes des deux copistes. La ponctuation est parfois trop sobre; un seul exemple: "[Seth doncques] en ensuivant le commandement de ses pere et mere ensemble la persuasion de l'archangele fist deux grandes tables [...] esqueles il escrivy la creation et vie de ses predecesseurs ensemble tout ce qu'il avoit veu et ö̈» (p. 108); il me semble qu'une virgule après «mere» et après «predecesseurs» éclarcirait le sens du passage.

Le texte est bien transcrit: je n'ai relevé qu'une faute (rugoreuse p. 81: lire rigoreuse). Les leçons qui interpellent à la lecture (par ex.: le douleur / la douleur à trois lignes de distance p. 96; Michil p. 94, partout ailleurs Michiel; les misteres futures p. 100, alors que seul le masculin est attesté dans les dictionnaires historiques) se trouvent bien dans le manuscrit fr. 1837 (numérisé dans Gallica ainsi que le manuscrit de l'Arsenal). L'attitude non interventionniste de l'éditrice me paraît parfois discutable, surtout dans les cas où une leçon meilleure est disponible dans les manuscrits de contrôle pour les parties communes: «Adam prist [Eve et Caÿm] et les mena en orient son habitation» (p. 98; A: en son h.). Un doute reste pour «ay esté [...] vaincue seconde fois» (p. 95; A E la s. f.), qui se représente plus loin «il luy commanda que tierce foiz il retournast» (p. 112): non enregistrée dans le DMF, cette forme aurait mérité soit un commentaire, soit une entrée dans le Glossaire. Parfaitement consciente du caractère hybride du texte qu'elle donne à lire (p. 57), S.M. n'oublie jamais de souligner dans les notes en pied de page les changements de texte de base. L'apparat, qui trouve place après le texte (pp.121-134), est malheureusement difficile à consulter; divisé en paragraphes, il ne renvoie à aucune autre subdivision: le lecteur intéressé à connaître les variantes fournies par les autres manuscrits se trouve obligé à le parcourir attentivement en entier pour retrouver la leçon qui l'intéresse (la présence de variantes n'étant même pas indiquée à l'intérieur du texte critique). Les notes au texte, appuyées pour l'essentiel sur l'édition critique de la Vita latine (par Jean-Pierre Pettorelli, Turnhout, 2012) se trouvent à la suite (pp. 135-150). Suivent l'Index des manuscrits mentionnés (pp. 151-152) et l'Index des noms propres (pp. 153-156). Le Glossaire tient compte fort opportunément des leçons qui se lisent dans la source latine, qui permet souvent de choisir l'acception à retenir; on aurait aimé savoir sur la base de quels dictionnaires historiques il a été établi (dans les quelques commentaires sur le lexique, pp. 22-24, le DMF n'est pas mentionné); et surtout y retrouver quelques mots de plus: anuyable (p. 80), anuyeuse (rapporté à la «vie», p. 110), appetible (p. 87), ascouter (p. 100 deux fois, 106), doleur masc. (p. 96), labeurs fém. (p. 106), parler transitif («ont parlé orguilleuses parolles», p. 109), psalme fém. (p. 117), (homme) de sang (p. 117), secondement (p. 93). En l'absence du commentaire linguistique, quelques formes verbales auraient pu être insérées: serra (fut. de seoir: «et alors il serra ou throne» p. 107), verront (fut. de venir, p. 106), voise (subjonctif P1 de aller, p. 102). Quelques traductions semblent imprécises: pour bouter hors (de), plutôt que 'pousser (hors de)' (p. 159), je proposerais 'chasser' («l'angele Cherubin bouta Adam et Eve hors de Paradis terrestre», p. 88). On est aussi surpris de trouver, sous l'entrée 
[croire] les formes de cuidier (cuident, cuidoit, cuidoyent) à côté de creoyent, creront, croiront, creans (p. 161).

8 Au-delà de ces quelques détails, on ne peut que saluer la parution de cette édition d'une œuvre presque inconnue de Colard Mansion, figure emblématique, de par la diversité même de sa production, de la deuxième moitié $d u \mathrm{xv}^{\mathrm{e}}$ siècle, résolument tournée vers la modernité tant par son activité de traducteur vers la langue française que par son passage de la copie à la main vers l'imprimerie. 